Groups Geom. Dyn. 7 (2013), 791-820

DOI $10.4171 / \mathrm{GGD} / 206$
Groups, Geometry, and Dynamics

(C) European Mathematical Society

\title{
Spectral properties of a class of random walks on locally finite groups
}

\author{
Alexander Bendikov, Barbara Bobikau and Christophe Pittet*
}

\begin{abstract}
We study some spectral properties of random walks on infinite countable amenable groups with an emphasis on locally finite groups, e.g. the infinite symmetric group $S_{\infty}$. On locally finite groups, the random walks under consideration are driven by infinite divisible distributions. This allows us to embed our random walks into continuous time Lévy processes. We obtain examples of fast/slow decays of return probabilities, a recurrence criterion, exact values and estimates of isospectral profiles and spectral distributions.
\end{abstract}

Mathematics Subject Classification (2010). 60B15, 62E10, 43A05.

Keywords. Random walk, locally finite group, ultra-metric space, infinite divisible distribution, Laplace transform, Köhlbecker transform, Legendre transform, return probability, spectral distribution, isospectral profile.

\section{Introduction}

We apply methods from analysis (Laplace, Köhlbecker, and Legendre transforms) and from geometric group theory (volume growth, isoperimetic and isospectral inequalities) to study the spectral properties of random walks on countable groups. Most of the results are about groups which are not finitely generated. All the random walks we consider are symmetric and invariant under left translations.

Notation. We use the following notation. For two non-negative functions defined on $\mathbb{R}_{+}$or on a subset $I$ of $\mathbb{R}_{+}$which is either a neighborhood of zero or of the infinity, we write:

- $f \asymp g$ if there are constants $a_{1}, a_{2}>0$ such that $a_{1} f(x) \leq g(x) \leq a_{2} f(x)$ for all $x \in I$ (factor equivalence),

- $f \stackrel{d}{\simeq} g$ if there are constants $b_{1}, b_{2}>0$ such that $f\left(b_{1} x\right) \leq g(x) \leq f\left(b_{2} x\right)$ for all $x \in I$ (dilatational equivalence),

${ }^{*}$ Research of A. Bendikov and B. Bobikau was supported by the Polish Government Scientific Research Fund, Grant N N201 371736. C. Pittet was supported by the CNRS. 
- $f \stackrel{d}{=} g$ if there are constants $a_{1}, a_{2}, b_{1}, b_{2}>0$ such that $a_{1} f\left(b_{1} x\right) \leq g(x) \leq$ $a_{2} f\left(b_{2} x\right)$ for all $x \in I$.

We also use the standard notation:

- $f \sim g$ at $a$ if $f(x) / g(x) \rightarrow 1$ at $a$.

An early result by Kesten [26] is that a countable group $G$ is amenable if and only if the spectral radius of the Markov operator associated to any symmetric irreducible random walk on $G$ equals 1 . In other words, a countable group is non-amenable if and only if the return probabilities of any symmetric irreducible random walk on it decay exponentially fast as time goes to infinity. (This equivalence generalizes to locally compact groups [6].) In Theorem 2.3 , we prove that any locally compact unimodular non-compact (hence any countable infinite) amenable group carries an irreducible symmetric random walk whose return probability decay is faster than any given sub-exponential function.

Recurrence criteria for random walks on countable infinitely generated groups have attracted much attention (see Chapter 4 below for a short description of contributions by Brofferio and Woess [7], Darling and Erdös [12], Dudly [14], Flato and Pitt [19], Kasymdzhanova [25], Molchanov and Nabil [18], Revuz [33], Chapter 9, Spitzer [36], Chapter 7), see also [5]. Recall that a group is locally finite if any of its finite subset generates a finite subgroup. Obviously, a group is countable locally finite if and only if it is a countable increasing union of finite subgroups. Any such group is amenable. It is not finitely generated if and only if the union is infinite and strictly increasing. A typical example is the group $S_{\infty}$ of permutations of the integers with finite supports. Lawler [27] has obtained a sufficient condition for recurrence on countable locally finite groups (see Proposition 4.1 below and [7], Proposition 1). In Proposition 4.2, we prove that Lawler's condition is also necessary for measures which are convex linear combinations of idempotent measures.

It is well known that on an infinite finitely generated group, any symmetric irreducible random walk returns to the origin at time $t$ with probability at most $t^{-1 / 2}$ (up to a constant rescaling factor). In other words, the slowest possible decay of return probabilities on an infinite finitely generated groups, is the one of the simple random walk on $\mathbb{Z}$. A short proof of this fact is obtained by applying Cheeger's inequality [13], Theorem 2.3, which implies a lower bound on the isospectral profile which we now define. Let $\mu$ be a symmetric irreducible probability measure on a countable group $G$ and let $P(f)=f * \mu$ be the corresponding right convolution operator on $L^{2}(G)$. The associated Laplace operator $\Delta=P-I$ is bounded, self-adjoint and $-\Delta$ is positive. The isospectral profile (also called the $L^{2}$-isoperimetric profile) of $(G, \mu)$ is the function

$$
\Lambda(v)=\min _{|\Omega| \leq v} \lambda_{1}(\Omega)
$$


where the minimum is taken over all finite subsets of $G$ of cardinality less or equal to $v$. Here,

$$
\lambda_{1}(\Omega)=\min _{\operatorname{supp}(f) \subset \Omega} \frac{(-\Delta f, f)}{\|f\|_{2}^{2}} .
$$

In contrast with finitely generated groups, there is no slowest decay of return probabilities on countable groups which are not finitely generated: in Proposition 4.3 we construct, on any infinite countable locally finite group, random walks with return probabilities whose decay is slower than any given positive function which goes to zero as time goes to infinity.

On a finitely generated group it is usually difficult (if not hopeless) to find an irreducible probability measure and to compute exact values of its isoperimetric profile or the spectral density driven by this measure. If $G$ is an infinite countable locally finite group, we can choose finite subgroups $G_{0} \subset G_{1} \subset \cdots$ of $G$ such that

$$
G=\bigcup_{k \geq 0} G_{k} .
$$

Any probability measure $\mu$ on $G$ can be represented as a convex linear combination

$$
\mu=\sum_{k=0}^{\infty} c_{k} \mu_{k}
$$

of probabilities $\mu_{k}$, each of which is supported by the finite subgroup $G_{k}$ (see Proposition 3.1). A natural choice for $\mu_{k}$ is the homogeneous probability measure on $G_{k}$ (i.e. the normalized Haar measure of $G_{k}$ ):

$$
\mu_{k}=m_{G_{k}} .
$$

The above representation of $\mu$ as a series of Haar measures is convenient for computations because if $v$ is a probability measure on a finite group $H$, then

$$
v * m_{H}=m_{H} * v=m_{H} .
$$

This in turn implies that $\mu$ is infinite divisible (see [17], IX.5, Theorem 2) and can be embed in a weakly continuous convolution semi-group $\left(\mu_{t}\right)$ of probability measures on $G$ (see Proposition 3.2). It allows us to compute exact values of the isospectral profile $\Lambda_{\mu}$ (Theorem 5.2 and Proposition 5.3).

Let $P$ be a right convolution operator defined by a symmetric probability measure on a countable group. Let $\lambda \rightarrow E_{\lambda}$ be the spectral resolution of the Laplacian $-\Delta=I-P$,

$$
-\Delta=\int_{0}^{\infty} \lambda d E_{\lambda} .
$$

Let $\delta_{e}$ denote the characteristic function of the identity element $e \in G$. We define the spectral distribution function $\lambda \rightarrow N(\lambda)$ as

$$
N(\lambda):=\left(E_{\lambda} \delta_{e}, \delta_{e}\right) .
$$


The asymptotic behavior of $N(\lambda)$, for $\lambda$ close to zero, for Laplace operators associated to simple random walks on finitely generated virtually nilpotent groups, can be deduced from Varopoulos results [28], Lemma 2.46. On finitely generated groups, and under some regularity assumption, the spectral distribution and the isospectral profile are related by the formula

$$
N(\lambda) \stackrel{d}{\simeq} \frac{1}{\Lambda^{-1}(\lambda)},
$$

(see [4]). In the case of infinite countable locally finite groups (they are of course never finitely generated), if the decay of the coefficients $c_{k}$ of the series of Haar measures $\left(\mu_{k}\right)$

$$
\mu=\sum_{k=0}^{\infty} c_{k} \mu_{k},
$$

is not too fast, namely, if there exists $\varepsilon>0$ such that

$$
\sum_{k>n} c_{k} \geq \varepsilon c_{n}
$$

for all $n \in \mathbb{N}$, then the same formula holds true (See Proposition 6.1).

\section{Convolution powers on unimodular groups}

Let $(G, m)$ be a locally compact, non-compact, unimodular group endowed with a left Haar measure $m$. Let $\left\{\mathfrak{B}_{k}\right\}_{k \in \mathbb{N}}$ be an increasing sequence of Borel subsets of $G$ such that $\left|\mathfrak{B}_{k}\right|:=m\left(\mathfrak{B}_{k}\right) \rightarrow \infty$ as $k \rightarrow \infty$.

With any sequence of positive reals $c=\left(c_{k}\right)_{k \in \mathbb{N}}$ such that $\sum_{k \in \mathbb{N}} c_{k}=1$ we associate the function

$$
x \rightarrow M(x)=\sum_{k \in \mathbb{N}} \frac{c_{k}}{\left|\mathfrak{B}_{k}\right|} \mathbf{1}_{\mathfrak{B}_{k}}(x) .
$$

Evidently $\mathfrak{B} \rightarrow M(\mathfrak{B})=\int_{\mathfrak{B}} M d m$ is a probability measure on $G$. Assume that all $\mathfrak{B}_{k}$ are symmetric and $\bigcup_{k} \mathfrak{B}_{k}$ generates a dense subgroup of $G$. Then $M$ is symmetric and supp $M$ generates a dense subgroup of $G$. Let $\mathfrak{R}_{M}: L^{2} \rightarrow L^{2}$ be the corresponding right-convolution operator $h \rightarrow h * M$. In general, $\left\|\mathfrak{L}_{M}\right\|_{L^{2} \rightarrow L^{2}} \leq 1$ and it is equal to 1 if and only if the group $G$ is amenable. On the other hand, let $\left\{X_{k}\right\}$ be i.i.d. random variables on $G$ with law $\mathbb{P}_{X_{1}}=M$ and let $S_{n}=X_{1} \cdot X_{2} \ldots X_{n}$ be the corresponding random walk on $G$. According to [6] the following characterization of $S_{n}$ via the norm of the convolution operator $\mathfrak{L}_{M}$ holds: For all relatively compact neighborhoods $V$ of the neutral element $e \in G$,

$$
\lim _{n \rightarrow \infty} \mathbb{P}\left(S_{2 n} \in V\right)^{1 / 2 n}=\left\|\mathfrak{L}_{M}\right\|_{L^{2} \rightarrow L^{2}} .
$$


In particular, if $G$ is amenable $\left\|\mathfrak{R}_{M}\right\|_{L^{2} \rightarrow L^{2}}=1$ and therefore

$$
\mathbb{P}\left(S_{2 n} \in V\right)=\exp (-n \cdot o(1)) \quad \text { as } n \rightarrow \infty .
$$

If the group $G$ is not amenable, then $\left\|\mathfrak{R}_{M}\right\|_{L^{2} \rightarrow L^{2}}<1$. This implies that the decay at infinity of the function $n \rightarrow \mathbb{P}\left(S_{2 n} \in V\right)$ is always exponential.

We claim that for any non-compact unimodular amenable group $G$, the decay of the function $n \rightarrow \mathbb{P}\left(S_{2 n} \in V\right)$ can be made as close as possible to the exponential one by an appropriate choice of the probability measure $M=\mathbb{P}_{X_{1}}$.

Observe that $M^{* n}(V) \leq\left\|M^{* n}\right\|_{\infty} m(V)$, hence to prove our claim it is enough to estimate the decay of the function $n \rightarrow\left\|M^{* n}\right\|_{\infty}$. We denote $\sigma_{k}=\sum_{i \leq k} c_{i}$ and $\sigma(k)=1-\sigma_{k}$. Let $\lambda \rightarrow \mathcal{N}(\lambda)$ be a right-continuous, non-decreasing stepfunction $\mathbb{R} \rightarrow \mathbb{R}_{+}$having jumps at the points $\lambda_{k}=\sigma(k)$ and taking values at these points $\mathcal{N}\left(\lambda_{k}\right)=1 /\left|\mathfrak{B}_{k}\right|$. Notice that $\lambda \rightarrow \mathcal{N}(\lambda)$ must be continuous at $\lambda=0$ and $\mathcal{N}(\lambda)=0$ for $\lambda \leq 0$. Later in the paper (see for example the proof of Proposition 4.2) we will consider functions of this kind arising as spectral distributions - as defined in the introduction - of explicit Laplace operators. When this is the case, we use the notation $N$ rather than $\mathcal{N}$. Following the paper [35] one can obtain the following result.

Proposition 2.1. In the notation introduced above the following inequality holds:

$$
\left\|M^{* n}\right\|_{\infty} \leq \int_{\mathbb{R}_{+}} e^{-n \sigma} d \mathcal{N}(\sigma), \quad n \in \mathbb{N} .
$$

We write $\mathcal{N}=e^{-\mathcal{M}}$ and introduce the following auxiliary transforms.

- The Köhlbecker transform of $\mathcal{M}$ :

$$
\mathcal{K}(\mathcal{M})(x):=-\log \left(\int_{0}^{\infty} e^{-x t} d e^{-\mathcal{M}(t)}\right) .
$$

- The Legendre transform of $\mathcal{M}$ :

$$
\mathscr{L}(\mathcal{M})(x):=\inf _{\tau>0}\{x \tau+\mathcal{M}(\tau)\} .
$$

Clearly, $\mathscr{L}(\mathcal{M}): \mathbb{R}_{+} \rightarrow \mathbb{R}_{+}$is a non-decreasing, concave function. In particular, it is continuous. For any non-decreasing, continuous function $\mathscr{F}: \mathbb{R}_{+} \rightarrow \mathbb{R}_{+}$, we define the conjugate Legendre transform of $\mathscr{F}$ as follows:

$$
\mathscr{L}^{*}(\mathcal{F})(x):=\sup _{\tau>0}\{-x \tau+\mathcal{F}(\tau)\} .
$$

Proposition 2.2. With the above notation, the following properties hold true.

(1) $\mathcal{K}(\mathcal{M})(x) \sim \mathscr{L}(\mathcal{M})(x)$ at $\infty$. 
(2) $\mathscr{L}\left(\mathscr{L}^{*}(\mathcal{F})\right) \geq \mathscr{F}$, and $\mathscr{L}\left(\mathscr{L}^{*}(\mathcal{F})\right)=\mathscr{F}$ if $\mathscr{F}$ is concave.

(3) For any continuous decreasing function $\mathcal{M}: \mathbb{R}_{+} \rightarrow \mathbb{R}_{+}$such that $\mathcal{M}(+0)=$ $+\infty$

$$
\mathscr{L}(\mathcal{M}) \asymp \mathcal{M} \circ(\mathcal{M} / \mathrm{id})^{-1} .
$$

(4) For any continuous function $\mathcal{F}: \mathbb{R}_{+} \rightarrow \mathbb{R}_{+}$such that $\mathscr{F}(+\infty)=+\infty$ and $\mathcal{F}(t)=o(t)$ at $\infty$, there exist two continuous decreasing functions $\mathcal{M}_{1}$, $\mathcal{M}_{2}: \mathbb{R}_{+} \rightarrow \mathbb{R}_{+}$, with $\mathcal{M}_{i}(+0)=+\infty, i=1,2$, such that

$$
\mathscr{L}\left(\mathcal{M}_{1}\right) / \mathscr{F} \rightarrow \infty \text { and } \mathscr{L}\left(\mathcal{M}_{2}\right) / \mathscr{F} \rightarrow 0 \text { at } \infty .
$$

Proof. The first statement is Lemma 3.2 in [3]. The second statement is a standard fact from convex analysis, see [34], Section 12. For the third statement see [4]. We prove the fourth statement: (a) Choose a continuous $\widetilde{\mathscr{F}}_{1}: \mathbb{R}_{+} \rightarrow \mathbb{R}_{+}$such that close to zero $\widetilde{\mathcal{F}}_{1}>c>0, \widetilde{\mathcal{F}}_{1} / \mathscr{F}_{\rightarrow} \rightarrow$ and $\widetilde{\mathcal{F}}_{1}(\tau)=o(\tau)$ at $\infty$. Put $\mathcal{M}_{1}=\mathscr{L}^{*}\left(\widetilde{\mathcal{F}}_{1}\right)$ and observe that $\mathcal{M}_{1}$ is convex, hence continuous. The second statement of Proposition 2.2 implies that

$$
\mathscr{L}\left(\mathcal{M}_{1}\right) / \mathscr{F} \geq \widetilde{\mathcal{F}}_{1} / \mathscr{F} \rightarrow \infty \quad \text { at } \infty .
$$

(b) Choose a concave $\widetilde{\mathscr{F}}_{2}: \mathbb{R}_{+} \rightarrow \mathbb{R}_{+}$such that near zero $\widetilde{\mathscr{F}}_{2}(t) \geq c>0$, $\widetilde{F}_{2}(t) / \mathscr{F}_{1}(t) \rightarrow 0$ and $\widetilde{\mathcal{F}}_{2}(\tau)=o(\tau)$ at $\infty$.

Put $\mathcal{M}_{2}=\mathscr{L}^{*}\left(\widetilde{\mathcal{F}}_{2}\right)$. The function $\mathcal{M}_{2}$ has the desired properties and according to the second statement of Proposition 2.2,

$$
\mathscr{L}\left(\mathcal{M}_{2}\right) / \mathscr{F}=\widetilde{\mathcal{F}}_{2} / \mathscr{F} \rightarrow 0 \quad \text { at } \infty .
$$

The proof is finished.

Theorem 2.3. Let $G$ be a locally compact non-compact group. Assume that $G$ is both unimodular and amenable. Let $\mathcal{F}: \mathbb{R}_{+} \rightarrow \mathbb{R}_{+}$be a non-decreasing function such that $\mathcal{F}(t)=o(t)$ at infinity. There exists a symmetric strictly positive probability density $M$ on $G$ such that

$$
-\log \left\|M^{* n}\right\|_{\infty} / \mathscr{F}(n) \rightarrow \infty \text { at } \infty .
$$

Proof. For $\mathscr{F}$ given, choose $\mathcal{M}$ such that $\mathscr{L}(\mathcal{M}) / \mathscr{F} \rightarrow \infty$ at $\infty$. See Proposition 2.2 (4). Choose an increasing sequence of Borel sets $\mathfrak{B}_{k} \subset G$ such that $\left|\mathfrak{B}_{k}\right| \rightarrow \infty$ and define a decreasing sequence $\{\sigma(k)\}$ from the equation $e^{-\mathcal{M}(\sigma(k))}=\left|\mathfrak{B}_{k}\right|^{-1}, k \gg 1$. Then define a sequence $\left\{c_{k}\right\}$ as follows: For $k \geq k_{0} \gg 1$ put $c_{k}=\sigma(k-1)-\sigma(k)$ and for $1 \leq k<k_{0}$ choose $c_{k}>0$ such that $\sum_{k=0}^{\infty} c_{k}=1$. Finally define

$$
M=\sum_{k=0}^{\infty} c_{k} \frac{1}{\left|\mathfrak{B}_{k}\right|} \mathbf{1}_{\mathfrak{B}_{k}},
$$

and a step-function $\mathcal{N}$ which is right-continuous, non-decreasing and has jumps at points $\lambda_{k}=\sigma(k)$ with values $\mathcal{N}\left(\lambda_{k}\right)=1 /\left|\mathfrak{B}_{k}\right|$. Write $\mathcal{N}=e^{-\widetilde{\mathcal{M}}}$. Clearly $\tilde{\mathcal{M}} \geq \mathcal{M}$ 
and therefore $\mathcal{K}(\tilde{\mathcal{M}}) \geq \mathcal{K}(\mathcal{M})$. Applying Proposition 2.1 we come to the desired conclusion

$$
\begin{aligned}
-\log \left\|M^{* n}\right\|_{\infty} / \mathscr{F}(n) & \geq \mathcal{K}(\tilde{\mathcal{M}}) / \mathscr{F}(n) \\
& \geq \mathcal{K}(\mathcal{M}) / \mathscr{F}(n) \sim \mathscr{L}(\mathcal{M}) / \mathscr{F}(n) \rightarrow \infty \quad \text { at } \infty .
\end{aligned}
$$

The proof is finished.

This proves the claim stated in the beginning of this section. Observe that the construction given in the proof of Theorem 2.3 can be used to build many examples of fast decaying convolution powers on locally compact non-compact unimodular amenable groups. For example, choose $l \geq 1$ and for $k \geq k_{0}$ big enough put

$$
\sigma(k)=\left(\log _{(l+1)}\left|\mathfrak{B}_{k}\right|\right)^{\frac{1}{v}} .
$$

Define $c_{k}>0$ such that $\sum_{k \geq 0} c_{k}=1$ and $c_{k}=\sigma(k-1)-\sigma(k)$ for $k \geq k_{0}+1$. Then the probability density $M$ defined by (2.1) satisfies

$$
\left\|M^{* n}\right\|_{\infty} \leq \exp \left(-\frac{c n}{\left(\log _{(l)} n\right)^{\frac{1}{v}}}\right) \quad \text { at } \infty,
$$

for some constant $c>0$.

\section{Convolution powers on locally finite groups}

Assume that the group $G$ under consideration has the following structure: there exists a strictly increasing sequence of finite subgroups $\left\{G_{k}\right\}$ of $G$ such that $G=\bigcup_{k} G_{k}$. We say that $G$ is locally finite. Evidently any such group is non-compact (in the discrete topology) unimodular and amenable.

Proposition 3.1. Let $G$ be a locally finite group. Any probability $\mu$ on $G$ can be represented as a convex linear combination

$$
\mu=\sum_{k=0}^{\infty} c_{k} \mu_{k}
$$

of probabilities $\mu_{k}$ each of which is supported by a finite subgroup $G_{k}$. The sequence $\left\{G_{k}\right\}$ increases and $G=\bigcup_{k} G_{k}$.

Proof. Indeed, if $|\operatorname{supp} \mu|=\infty$, choose any increasing sequence $\left\{G_{k}\right\}$ of finite subgroups of $G$ such that $\mu\left(G_{0}\right)>0$. Then $\mu\left(G \backslash G_{k}\right)>0$ for all $k>0$ and let

$$
\mu_{k}:=\left(\sum_{l=0}^{k} \frac{\mu\left(G_{l} \backslash G_{l-1}\right)}{\mu\left(G \backslash G_{l-1}\right)}\right)^{-1}\left(\sum_{l=0}^{k} \frac{\mathbf{1}_{G_{l} \backslash G_{l-1}}}{\mu\left(G \backslash G_{l-1}\right)}\right) \mu
$$


and

$$
c_{k}:=\mu\left(G_{k} \backslash G_{k-1}\right)\left(\sum_{l=0}^{k} \frac{\mu\left(G_{l} \backslash G_{l-1}\right)}{\mu\left(G \backslash G_{l-1}\right)}\right),
$$

where $G_{-1}:=\emptyset$. The proof is finished.

In [7] a few different choices of $\left(\mu_{k}, c_{k}\right)$ (called shuffling models) are presented. In the present paper we consider the following special cases: Let $m_{k}$ be the normalized Haar measure on $G_{k}$. Let $c=\left\{c_{k}\right\}$ be a sequence of strictly positive reals such that $\sum_{k} c_{k}=1$. Define a probability measure $\mu=\mu(c)$ on $G$ by

$$
\mu=\sum_{k} c_{k} m_{k}
$$

Let $m$ be the counting measure on $G$ and $L^{2}=L^{2}(G, m)$. Define on $L^{2}$ the following linear operators $P_{k} f=f * m_{k}$ and $P f=f * \mu$. Clearly $P_{k}$ and $P$ are bounded symmetric operators and

$$
P=\sum_{k=0}^{\infty} c_{k} P_{k}
$$

Let us compute $P^{n}$. Since $m_{l} * m_{k}=m_{\max }(l, k)$,

$$
P^{n}=\sum_{k=0}^{\infty} a_{k} P_{k},
$$

where

$$
a_{k}=\left\{\begin{array}{l}
\left(c_{0}+\cdots+c_{k}\right)^{n}-\left(c_{0}+\cdots+c_{k-1}\right)^{n}, \quad k \geq 1, \\
a_{0}=c_{0}^{n} .
\end{array}\right.
$$

From these equations we obtain

$$
c_{k}=\left\{\begin{array}{l}
\left(a_{0}+\cdots+a_{k}\right)^{\frac{1}{n}}-\left(a_{0}+\cdots+a_{k-1}\right)^{\frac{1}{n}}, \quad k \geq 1, \\
c_{0}=a_{0}^{\frac{1}{n}} .
\end{array}\right.
$$

In particular, the symmetric non-negative definite operator $P^{\frac{1}{m}}$, defined via spectral theory, has the following representation:

$$
P^{\frac{1}{m}}=\sum_{k=0}^{\infty}\left(\left(c_{0}+\cdots+c_{k}\right)^{\frac{1}{m}}-\left(c_{0}+\cdots+c_{k-1}\right)^{\frac{1}{m}}\right) P_{k} .
$$

As a consequence of these two observations we obtain:

$$
P^{\frac{n}{m}}=\sum_{k=0}^{\infty}\left(\left(c_{0}+\cdots+c_{k}\right)^{\frac{n}{m}}-\left(c_{0}+\cdots+c_{k-1}\right)^{\frac{n}{m}}\right) P_{k} .
$$


Let now $t \in \mathbb{R}_{+}$and $r_{n} \in \mathbb{Q}_{+}$be a sequence of rationals such that $r_{n} \rightarrow t$. Then, by spectral theory, $P^{r_{n}} \rightarrow P^{t}$ strongly. Hence, passing to the limit in both sides of the equation (3.1) we obtain

$$
P^{t}=\sum_{k=0}^{\infty}\left(\left(c_{0}+\cdots+c_{k}\right)^{t}-\left(c_{0}+\cdots+c_{k-1}\right)^{t}\right) P_{k} .
$$

The above facts are crucial for our purposes. We summarize them in the following proposition.

Proposition 3.2. The measure $\mu=\mu(c)$ is infinite divisible. In particular, there exists a weakly continuous convolution semigroup $\left(\mu_{t}\right)_{t>0}$ of probability measures on $G$ such that $\mu=\mu_{1}$. Moreover the following representation holds:

$$
\mu_{t}=\sum_{k=0}^{\infty} c_{k}(t) m_{k}
$$

where

$$
c_{k}(t)=\left\{\begin{array}{l}
\left(c_{0}+\cdots+c_{k}\right)^{t}-\left(c_{0}+\cdots+c_{k-1}\right)^{t}, \quad k \geq 1 \\
c_{0}(t)=c_{0}^{t}
\end{array}\right.
$$

\section{Recurrence criterion}

The problem of recurrence of random walks on countable abelian groups with finite number of generators has been investigated in details in the book of Spitzer [36], Chapter 7. Dudley [14] proved that each countable abelian group which does not contain a subgroup isomorphic $\mathbb{Z}^{3}$ admits a irreducible recurrent random walk. The proof of Dudley is not constructive, hence it is very desirable to find some explicit construction of recurrent random walks on such groups. This construction has been done in the works of S. Molchanov and his collaborators [18], [25] for the following groups: $G=\mathbb{Z}[1 / p]$, the group of all rational numbers of the form $r=\frac{k}{p^{m}}$ where $k, p>1$ and $m \geq 0$ are integers, and $G=\Gamma_{p}=\mathbb{Z}^{k} \times(\mathbb{Z} / p \mathbb{Z})^{(\infty)}$, with $k=0,1,2$. They obtained sufficient conditions for the recurrence and also necessary ones which are very close to each other. Brofferio and Woess [7] proved recurrence criteria for certain card shuffling models, that is, random walks on the infinite symmetric group $S_{\infty}=\bigcup_{n>1} S_{n}$. One of these models has been considered previously by Lawler [27]. He obtained a very general sufficient condition for recurrence:

Proposition 4.1. A random walk on $G=\bigcup_{n \geq 0} G_{n}$ with law $\mu$ is recurrent if

$$
\sum_{n=1}^{\infty} \frac{1}{\left|G_{n}\right|\left(1-\mu\left(G_{n}\right)\right)}=\infty
$$


Special cases of Proposition 4.1 had been proved previously by Flatto and Pitt [19] (each $G_{n}$ is cyclic, or $G$ is the direct sum of finite abelian groups), and before that by Darling and Erdős [12] ( $G$ is the direct sum of infinitely many copies of the group of order two). See also [2].

If $\mu=\mu(c)$ is defined as in the proof of Proposition 3.1, we show that Lawler's above condition is also necessary.

Proposition 4.2. A random walk on $G$ with law $\mu=\mu(c)$ is recurrent if and only if the following condition holds:

$$
\sum_{n=0}^{\infty} \frac{1}{\left|G_{n}\right|\left(1-\mu\left(G_{n}\right)\right)}=\infty
$$

Proof. Let $N(\lambda)$ be the spectral distribution function associated to $\mu=\mu(c)$ as defined in the introduction. It is easy to see that $N(\lambda)$ is a right-continuous, nondecreasing step-function having jumps at the points $\lambda_{k}=\sigma(k)$, where $\sigma(k)=$ $\sum_{i>k} c_{i}$, and $N\left(\lambda_{k}\right)=1 /\left|G_{k}\right|$. By Proposition 3.2,

$$
\mu^{* n}(e)=\int_{0}^{\infty}(1-\lambda)^{n} d N(\lambda)
$$

Since the measure $\mathfrak{B} \rightarrow \int_{\mathfrak{B}} d N$ is supported by the interval $[0, \sigma(0)] \subset[0,1]$, there exists $\delta>1$ such that, for all $n \in \mathbb{N}$,

$$
\int_{0}^{\infty} e^{-n \delta \lambda} d N(\lambda) \leq \int_{0}^{\infty}(1-\lambda)^{n} d N(\lambda) \leq \int_{0}^{\infty} e^{-n \lambda} d N(\lambda) .
$$

Next we use a well-known criterion of recurrence: a random walk with law $\mu$ is recurrent if and only if

$$
\sum_{n} \mu^{* n}(e)=\infty
$$

Since

$$
\mu^{* n}(e) \stackrel{d}{\simeq} \int_{0}^{\infty} e^{-n \lambda} d N(\lambda)
$$

we easily transform this criterion into the property

$$
\sum_{k \geq 0} \frac{1}{\left|G_{k}\right| \sigma(k)} \asymp \int_{0}^{\infty} \frac{d N(\lambda)}{\lambda} \asymp \sum_{n} \mu^{* n}(e)=\infty .
$$

Finally, observe that $1-\mu\left(G_{n}\right) \sim \sigma(n)$. This finishes the proof.

It is well known that if a locally compact non-compact group $G$ is compactly generated (for example if $G$ is infinite and finitely generated), the upper rate of decay, among all functions defined by

$$
n \rightarrow\left\|\mu^{* 2 n}\right\|_{\infty}
$$


where $\mu$ is a symmetric probability density whose support generates $G$, is realized

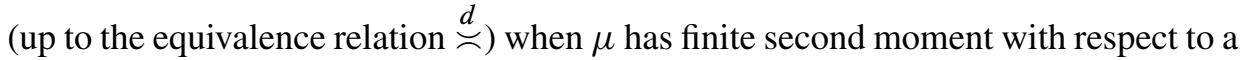
word metric defined by a compact symmetric generating set. This rate is a geometric invariant of the group $G$. See for instance [4], [30], [31]. In particular, let $G$ be an abelian non-compact compactly generated group. By the structure theory [24], Theorem 9.8,

$$
G \cong \mathbb{R}^{l} \times \mathbb{Z}^{m} \times K, \quad l+m>0,
$$

where $K$ is a compact group. Then, for any symmetric probability density $\mu: G \rightarrow$ $\mathbb{R}_{+}$whose support generates $G$, we must have

$$
\left\|\mu^{* 2 n}\right\|_{\infty} \leq C n^{-(l+m) / 2} \text { at } \infty .
$$

Let $\{X(n)\}_{n \geq 0}$ be the random walk on $G$ with law $\mu$. Assume that $\{X(n)\}$ is recurrent. Y. Guivarc'h asked the following question: How slow may the function $n \rightarrow \mathbb{P}(X(2 n)=e \mid X(0)=e)$ decay at infinity? The next proposition shows that if $G$ is locally compact but not compactly generated, the decay of the function $n \rightarrow\left\|\mu^{* 2 n}\right\|_{\infty}$ may be as slow as possible.

Proposition 4.3. Let $G$ be an infinite countable locally finite group and $\mathcal{F}: \mathbb{R}_{+} \rightarrow$ $\mathbb{R}_{+}$be a non-decreasing continuous function such that $\mathscr{F}(t)=o(t)$ and $\mathscr{F}(t) \rightarrow \infty$ at infinity. There exists a symmetric strictly positive probability density $\mu$ on $G$ such that

$$
-\log \mu^{* n}(e) / \mathcal{F}(n) \rightarrow 0 \quad \text { at } \infty,
$$

(compare with Theorem 2.3).

Proof. Let $G=\bigcup_{k=1}^{\infty} G_{k}$, where $\{e\}=G_{0} \subset G_{1} \subset \cdots \subset G_{k} \subset \cdots$ are finite subgroups of $G$. Let $\mu=\mu(c)$ for some $c=\left(c_{k}\right)$. Then by (4.1),

$$
\mu^{* n}(e) \geq \int_{0}^{\infty} e^{-\delta n \lambda} d N(\lambda)
$$

for some $\delta>1$. Proceeding as in the proof of Theorem 2.3 but choosing $N(\lambda) \geq$ $e^{-\mathcal{M}(\delta \lambda)}$ with $\mathcal{M}$ continuous and decreasing, and such that

$$
\mathscr{L}(\mathcal{M}) / \mathscr{F} \rightarrow 0 \text { at } \infty,
$$

we can find $c=\left(c_{k}\right)$ and hence $\mu=\mu(c)$ such that $c_{k}>0$ and

$$
\mu^{* n}(e) \geq \int_{0}^{\infty} e^{-n \delta \lambda} d N(\lambda) \geq \int_{0}^{\infty} e^{-n \lambda} d e^{-\mathcal{M}(\lambda)} .
$$

Therefore as $n \rightarrow \infty$ we obtain

$$
-\log \mu^{* n}(e) / \mathscr{F}(n) \leq \mathcal{K}(\mathcal{M}) / \mathscr{F}(n) \sim \mathscr{L}(\mathcal{M}) / \mathscr{F}(n) \rightarrow 0 .
$$

The proof of the proposition is finished. 


\section{5. $L^{2}$-isospectral profile}

Let $P$ and $\Delta=P-I$ be the transition operator and the Laplacian defined by the probability measure $\mu=\mu(c)$. For any subset $U \subset G$ we define the truncated operators $P_{U}$ and $\Delta_{U}$ by

$$
P_{U} f(x)=\mathbf{1}_{U}(x) P\left(\mathbf{1}_{U} f\right)(x)
$$

and

$$
\Delta_{U} f(x)=\mathbf{1}_{U}(x) \Delta\left(\mathbf{1}_{U} f\right)(x) .
$$

Let $m_{U}$ be the counting measure on $U$ and $L^{2}(U)=L^{2}\left(U, m_{U}\right)$. Evidently, the operators $P_{U}$ and $-\Delta_{U}$ can be regarded as operators on $L^{2}(U)$, each of which is a bounded and symmetric, and $-\Delta_{U}$ is non-negative definite. Moreover, $\Delta_{U}=$ $P_{U}-I$. If $U$ is a finite set, $L^{2}(U)$ is finite dimensional and its dimension $n$ is equal to $|U|$. Therefore the spectrum $\operatorname{Spec}_{L^{2}}\left(-\Delta_{U}\right)$ of $-\Delta_{U}$ consists of a finite number of points $0<\lambda_{1}(U) \leq \cdots \leq \lambda_{n}(U)<1$, repeated according to their multiplicity.

We define the $L^{2}$-isospectral profile $\lambda \rightarrow \Lambda(\lambda)$ by

$$
\Lambda(\lambda):=\inf _{U:|U| \leq \lambda} \lambda_{1}(U) .
$$

For any function $0<\Lambda_{*} \leq \Lambda$ the following Faber-Krahn type inequality holds true:

$$
\lambda_{1}(U) \geq \Lambda_{*}(|U|), \quad \text { for any finite } U \subset G .
$$

In the general setting of Markov generators this inequality was introduced in [1] (see also [20], [21]) to investigate various aspects of the heat kernel behavior. In particular, it was proved in [21] that under some regularity assumptions on $\Lambda_{*}$, the Faber-Krahn type inequality is equivalent to the heat kernel estimate

$$
\sup _{x, y \in G} h(t ; x, y) \leq \frac{1}{\Phi(t)} \quad \text { for all } t>0,
$$

where the functions $\Lambda_{*}$ and $\Phi$ are related by

$$
t=\int_{0}^{\Phi(t)} \frac{d \lambda}{\lambda \Lambda_{*}(\lambda)}
$$

See [8], [9], [10], [11], [29] and [30].

The main aim of this section is to obtain a lower bound for the function $\lambda \rightarrow \Lambda(\lambda)$ in terms of the sequences $\left\{c_{k}\right\}$ and $\left\{\left|G_{k}\right|\right\}$. Under certain regularity assumptions on $\left\{c_{k}\right\}$ we will obtain two-sided bounds for $\Lambda$ which are comparable in the sense of dilatational equivalence. See Theorem 5.2 and Theorem 5.7 below.

If $a, b$ are positive reals $a \wedge b$ denotes their minimum. We define the function $\mathcal{T}: \mathbb{R}_{+} \rightarrow \mathbb{R}_{+}$as follows:

$$
\mathcal{T}(u):=1-\sum_{i=0}^{\infty} c_{i}\left(1 \wedge \frac{u}{\left|G_{i}\right|}\right) .
$$


The next proposition easily follows from the very definition of the function $u \rightarrow$ $\mathcal{T}(u)$.

Proposition 5.1. The following properties hold true.

(1) $\mathcal{T}$ is a continuous function.

(2) $\mathcal{T}$ strictly decreases to zero at infinity.

(3) $\mathcal{T}$ is convex.

(4) For $\left|G_{k}\right| \leq u<\left|G_{k+1}\right|$,

$$
\frac{1}{2} \sigma(k+1)<\mathcal{T}(u)<\sigma(k) .
$$

Theorem 5.2. For any finite set $U \subset G$, the following inequality holds:

$$
\lambda_{1}(U) \geq \mathcal{T}(|U|) .
$$

In particular, for any $\lambda \geq 1$,

$$
\Lambda(\lambda) \geq \mathcal{T}(\lambda)
$$

Proof. Choose a finite set $U \subset G$, a function $f$ such that $\operatorname{supp}(f) \subset U$, and write

$$
\begin{aligned}
\left(f, P_{i} f\right) & =\sum_{x \in U} f(x) P_{i} f(x) \\
& =\sum_{x, y \in U} f(x) f(y) m_{i}\left(y^{-1} x\right) \\
& =\frac{1}{\left|G_{i}\right|} \sum_{x, y \in U: y^{-1} x \in G_{i}} f(x) f(y) .
\end{aligned}
$$

Let $\left[G: G_{i}\right]$ be the set of all co-sets $A=a G_{i}, a \in G$. Then

$$
\begin{aligned}
\sum_{x, y \in U: y^{-1}} f(x) f(y) & =\sum_{A \in\left[G: G_{i}\right]} \sum_{x, y \in A \cap U} f(x) f(y) \\
& =\sum_{A \in\left[G: G_{i}\right]}\left(\sum_{x \in A \cap U} f(x)\right)^{2} \\
& \leq \sum_{A \in\left[G: G_{i}\right]}|A \cap U| \sum_{x \in A \cap U} f(x)^{2} \\
& \leq \max _{A \in\left[G: G_{i}\right]}|A \cap U| \sum_{A \in\left[G: G_{i}\right]} \sum_{x \in A \cap U} f(x)^{2} \\
& =\max _{A \in\left[G: G_{i}\right]}|A \cap U|\|f\|_{L^{2}(U)}^{2} \\
& \leq\left(\left|G_{i}\right| \wedge|U|\right)\|f\|_{L^{2}(U)}^{2} .
\end{aligned}
$$


Hence, for any $i=0,1,2, \ldots$, and any finite set $U \subset G$ the operator norm of the truncated operator $P_{i, U}$ can be estimated as follows:

$$
\begin{aligned}
\left\|P_{i, U}\right\| & =\sup \left\{\left(f, P_{i} f\right): \operatorname{supp}(f) \subseteq U,\|f\|_{L^{2}}=1\right\} \\
& \leq \frac{1}{\left|G_{i}\right|}\left(\left|G_{i}\right| \wedge|U|\right)=\left(1 \wedge \frac{|U|}{\left|G_{i}\right|}\right) .
\end{aligned}
$$

It follows that

$$
\left\|P_{U}\right\| \leq \sum_{i=0}^{\infty} c_{i}\left\|P_{i, U}\right\| \leq \sum_{i=0}^{\infty} c_{i}\left(1 \wedge \frac{|U|}{\left|G_{i}\right|}\right) .
$$

With this inequality in hands the computations of $\lambda_{1}(U)$ become straightforward:

$$
\begin{aligned}
\lambda_{1}(U) & =\min \left\{\left(-\Delta_{U} f, f\right): \operatorname{supp}(f) \subseteq U,\|f\|_{L^{2}}=1\right\} \\
& =\min \left\{1-\left(P_{U} f, f\right): \operatorname{supp}(f) \subseteq U,\|f\|_{L^{2}}=1\right\} \\
& =1-\max \left\{\left(P_{U} f, f\right): \operatorname{supp}(f) \subseteq U,\|f\|_{L^{2}}=1\right\} \\
& =1-\left\|P_{U}\right\| \\
& \geq 1-\sum_{i=0}^{\infty} c_{i}\left(1 \wedge \frac{|U|}{\left|G_{i}\right|}\right)=\mathcal{T}(|U|) .
\end{aligned}
$$

Since $\lambda \rightarrow \mathcal{T}(\lambda)$ is a decreasing function, we obtain

$$
\Lambda(\lambda)=\inf \left\{\lambda_{1}(U):|U| \leq \lambda\right\} \geq \mathcal{T}(\lambda) .
$$

The proof is finished.

Proposition 5.3. For any $k=0,1,2, \ldots$,

$$
\lambda_{1}\left(G_{k}\right)=\mathcal{T}\left(\left|G_{k}\right|\right) .
$$

In particular, $\frac{1}{2} \sigma(k)<\lambda_{1}\left(G_{k}\right)<\sigma(k)$.

Proof. Since $G_{k}$ is a subgroup of $G$, one can regard $P_{G_{k}}$ as a convolution operator on $G_{k}$. Indeed, for $x \in G_{k}$ and $f$ such that $\operatorname{supp}(f) \subseteq G_{k}$ we have

$$
P_{G_{k}} f(x)=\int_{G_{k}} f(x y) d \mu(y)=\int_{G_{k}} f(x y) d \mu_{G_{k}}(y)=f(x) * \mu_{G_{k}},
$$

where $\mu_{G_{k}}$ is the restriction of the probability measure $\mu$ on $G_{k}$. We have

$$
\begin{aligned}
\mu_{G_{k}} & =\sum_{i=0}^{\infty} c_{i} m_{i, G_{k}} \\
& =\sum_{i=0}^{k-1} c_{i} m_{i}+\sum_{i=k}^{\infty} c_{i} \frac{\left|G_{k}\right|}{\left|G_{i}\right|} m_{k} \\
& =\sum_{i=0}^{k-1} c_{i} m_{i}+\left(\sum_{i=k}^{\infty} c_{i} \frac{\left|G_{k}\right|}{\left|G_{i}\right|}\right) m_{k} .
\end{aligned}
$$


In particular, since $\left|G_{k}\right| /\left|G_{i}\right| \leq 1 / 2$, for $i>k$,

$$
\mu_{G_{k}}(1)=\sum_{i=0}^{k-1} c_{i}+\left(\sum_{i=k}^{\infty} c_{i} \frac{\left|G_{k}\right|}{\left|G_{i}\right|}\right) \leq \sum_{i=0}^{k} c_{i}+\frac{1}{2} \sum_{i=k+1}^{\infty} c_{i}<1 .
$$

Since for any finite group (more generally, any amenable group) the norm of the convolution operator equals the variation of the corresponding measure, we have

$$
\left\|P_{G_{k}}\right\|=\mu_{G_{k}}(1) .
$$

It follows that

$$
\lambda_{1}\left(G_{k}\right)=1-\left\|P_{G_{k}}\right\|=1-\sum_{i \leq k} c_{i}-\sum_{i>k} c_{i} \frac{\left|G_{k}\right|}{\left|G_{i}\right|}=\mathcal{T}\left(\left|G_{k}\right|\right) .
$$

We also have

$$
\lambda_{1}\left(G_{k}\right)=\sum_{i>k}\left(1-\frac{\left|G_{k}\right|}{\left|G_{i}\right|}\right) c_{i} .
$$

Since $\left|G_{k}\right| /\left|G_{i}\right| \leq 1 / 2$ for all $i>k$, we obtain

$$
\frac{1}{2} \sum_{i>k} c_{i}<\lambda_{1}\left(G_{k}\right)<\sum_{i>k} c_{k}
$$

The proof is finished.

According to Theorem 5.2 the function $u \rightarrow \mathcal{T}(u)$ is a lower bound for the function $u \rightarrow \Lambda(u)$. Following ideas of Følner (see e.g. [11], [15], [32]) we will give an upper bound for the function $u \rightarrow \Lambda(u)$.

Definition 5.4. For $n \in \mathbb{N}$ set

$$
k(n):=\min \left\{k: \lambda_{1}\left(G_{k}\right) \leq 1 / n^{2}\right\} .
$$

Let $v \rightarrow F(v)$ be the continuous piecewise linear function such that $F(n)=\left|G_{k(n)}\right|$. Observe that $v \rightarrow F(v)$ is a strictly increasing continuous function. Hence the inverse $v \rightarrow F^{-1}(v)$ exists in the usual sense. We define the function $\Lambda_{F}:(1,+\infty) \rightarrow \mathbb{R}_{+}^{1}$ by

$$
\Lambda_{F}(v):=\left(F^{-1}(v)-1\right)^{-2} .
$$

Proposition 5.5. The following inequality holds true:

$$
\Lambda(v) \leq \Lambda_{F}(v) \text { for all } v>1 .
$$


Proof. Let $\Omega_{n}:=G_{k(n)}$ and $\omega_{v}:=\Omega_{\left[F^{-1}(v)\right]}$. We have

$$
\left|\omega_{v}\right|=\left|\Omega_{\left[F^{-1}(v)\right]}\right|=F\left(\left[F^{-1}(v)\right]\right) \leq v .
$$

Using the definition of $k(n)$ we obtain

$$
\lambda_{1}\left(\omega_{v}\right)=\lambda_{1}\left(\Omega_{\left[F^{-1}(v)\right]}\right) \leq\left(F^{-1}(v)-1\right)^{-2}=\Lambda_{F}(v) .
$$

We conclude that

$$
\Lambda(v)=\inf \left\{\lambda_{1}(U):|U| \leq v\right\} \leq \lambda_{1}\left(\omega_{v}\right) \leq \Lambda_{F}(v) .
$$

The proof is finished.

The following regularity condition will play a crucial role in our further considerations.

(A) There exists $\lambda>0$ such that

$$
c_{k} \leq \lambda \sigma(k) \text { for all } k \in \mathbb{N},
$$

or equivalently,

$$
\sigma(k-1) \leq(1+\lambda) \sigma(k) \quad \text { for all } k \in \mathbb{N} .
$$

It is easy to see that for $c_{k}=\exp \left(-k^{p}\right)$ condition (A) holds if and only if $p \leq 1$.

Proposition 5.6. Assume that condition (A) holds. Then there exists $c>0$ such that

$$
\Lambda_{F}(v) \leq c \mathcal{T}(v) \text { for any } v>1 .
$$

Proof. For any $n \geq 1$ and $n \leq v \leq n+1$, we have

$$
\mathcal{T}(F(v)) \geq \mathcal{T}(F(n+1))=\mathcal{T}\left(\left|G_{k(n+1)}\right|\right)=\lambda_{1}\left(G_{k(n+1)}\right) \geq \frac{1}{2} \sigma(k(n+1)) .
$$

Due to our assumption

$$
\sigma(k(n+1)) \geq c_{1} \sigma(k(n+1)-1)
$$

for some $c_{1}>0$, hence

$\mathcal{T}(F(v)) \geq \frac{c_{1}}{2} \sigma(k(n+1)-1) \geq \frac{c_{1}}{2} \lambda_{1}\left(G_{k(n+1)-1}\right) \geq \frac{c_{1}}{2} \frac{1}{(n+1)^{2}} \geq \frac{c_{1}}{4} \frac{1}{v^{2}} \geq \frac{c_{2}}{v^{2}}$

for some $c_{2}>0$. Since $u \rightarrow F(u)$ is strictly increasing and since $F(\infty)=\infty$, there exists $c_{3}>0$ such that

$$
\mathcal{T}(u) \geq \frac{c_{2}}{\left(F^{-1}(u)\right)^{2}} \geq \frac{c_{3}}{\left(F^{-1}(u)-1\right)^{2}}=c_{3} \Lambda_{F}(u) .
$$

The proof is finished. 
Theorem 5.7. Assume that condition (A) holds. Then there exists $c>0$ such that

$$
\mathcal{T}(u) \leq \Lambda(u) \leq c \mathcal{T}(u) \text { for any } u>1,
$$

that is, $\Lambda \asymp \mathcal{T}$ at $\infty$.

Proof. We always have

$$
\Lambda_{F}(u) \geq \Lambda(u) \geq \mathcal{T}(u) \quad \text { for any } u>1 .
$$

Assuming that the condition (A) holds, we apply Proposition 5.6 and obtain:

$$
\Lambda_{F}(u) \leq c \mathcal{T}(u) \text { for any } u>1 .
$$

The proof is finished.

Proposition 5.8. Let $\phi, v: \mathbb{R}_{+} \rightarrow \mathbb{R}_{+}$be two continuous strictly monotone functions such that $\phi(k) \asymp \sigma(k)$ and $v(k) \asymp\left|G_{k}\right|$ at $\infty$. Then under condition (A)

$$
\mathcal{T} \asymp \phi \circ v^{-1} \text { and } \Lambda \stackrel{d}{\asymp} \phi \circ v^{-1} \text { at } \infty \text {. }
$$

Proof. Since $\Lambda \asymp \mathcal{T}$ we work with $\mathcal{T}$. According to Proposition 5.1 (4):

$$
\frac{1}{2} \sigma(k+1)<\mathcal{T}(u)<\sigma(k) \text { for }\left|G_{k}\right| \leq u<\left|G_{k+1}\right| .
$$

Hence for $u, k$ as above and for some $b_{1}, b_{2}>0$,

$$
k \leq v^{-1}\left(\frac{u}{b_{1}}\right), \quad k+1>v^{-1}\left(\frac{u}{b_{2}}\right) .
$$

It follows that for some $b_{3}, b_{4}>0$,

$$
\mathcal{T}(u)<\sigma(k) \leq(1+\lambda) \sigma(k+1) \leq b_{3}(1+\lambda) \phi(k+1) \leq b_{3}(1+\lambda) \phi \circ v^{-1}\left(\frac{u}{b_{2}}\right)
$$

and

$$
\mathcal{T}(u)>\frac{1}{2} \sigma(k+1) \geq \frac{1}{2(1+\lambda)} \sigma(k) \geq \frac{b_{4}}{2(1+\lambda)} \phi(k) \geq \frac{b_{4}}{2(1+\lambda)} \phi \circ v^{-1}\left(\frac{u}{b_{1}}\right) .
$$

Notice that the constants $b_{1}, b_{2}, b_{3}, b_{4}>0$ come from the relations $\phi(k) \asymp \sigma(k)$ and $v(k) \asymp\left|G_{k}\right|$. The proof is finished.

Example 5.9. 1) Let $G=\bigcup_{n=0}^{\infty} G_{n}, G_{n}=(\mathbb{Z} / 2 \mathbb{Z})^{n}$. Then $\left|G_{n}\right|=2^{n}$ and we can choose $v(x)=2^{x}, x \geq 0$. Proposition 5.8 yields the following result: Under condition (A), for any $\phi \asymp \sigma$ at $\infty$,

$$
\Lambda(\tau) \asymp \phi \circ \log \left(\tau^{\gamma}\right) \text { at } \infty, \text { where } \gamma=1 / \log 2 .
$$

In particular, we have 
(1.1) If $c_{k} \asymp q^{k}, 0<q<1$, then $\sigma(k) \asymp q^{k}, k \in \mathbb{Z}_{+}$. Hence,

$$
\Lambda(\tau) \asymp \tau^{-A \gamma} \quad \text { at } \infty \text {, where } A=\log \frac{1}{q} \text {. }
$$

(1.2) If $c_{k} \asymp k^{-p}, p>1$, then $\sigma(k) \asymp k^{-p+1}, k \in \mathbb{Z}_{+}$. Hence,

$$
\Lambda(\tau) \asymp(\log \tau)^{1-p} \text { at } \infty .
$$

(1.3) If $c_{k} \asymp 1 /\left(k \cdot \log k \cdot \log _{(2)} k \cdots\left(\log _{(n)} k\right)^{p}\right), p>1$, then

$$
\sigma(k) \asymp\left(\log _{(n)} k\right)^{1-p}, \quad k \in \mathbb{Z}_{+} .
$$

Hence,

$$
\Lambda(\tau) \asymp\left(\log _{(n+1)} \tau\right)^{1-p} \text { at } \infty .
$$

2) Let $G=\bigcup_{n=0}^{\infty} G_{n}, G_{n}=S_{n}$, be the infinite symmetric group, i.e. the group of all finite permutations of the set $\mathbb{N}=\{1,2, \ldots\}$. We have $\left|G_{n}\right|=n !$. Hence we can choose $v(x)=\Gamma(x+1)$, where $\Gamma(x)$ is the gamma function

$$
\Gamma(x)=\int_{0}^{\infty} t^{x-1} e^{-t} d t .
$$

By Stirling's formula [16], (9.15),

$$
\Gamma(1+x)=\sqrt{2 \pi x}\left(\frac{x}{e}\right)^{x}\left(1+O\left(\frac{1}{x}\right)\right) .
$$

Let $\phi: \mathbb{R}_{+} \rightarrow \mathbb{R}_{+}$be any continuous decreasing function such that $\phi(k) \asymp \sigma(k)$ at $\infty$. Proposition 5.8 shows that under condition (A),

$$
\Lambda \asymp \phi \circ \Gamma^{-1} \text { at } \infty \text {. }
$$

Definition 5.10. The upper order $\rho^{*}(f)$ and lower order $\rho_{*}(f)$ of a positive function $f$ are defined by

$$
\rho^{*}(f):=\limsup _{x \rightarrow \infty} \frac{\log f(x)}{\log x}, \quad \rho_{*}(f):=\liminf _{x \rightarrow \infty} \frac{\log f(x)}{\log x} .
$$

If $\rho^{*}(f)=\rho_{*}(f)<\infty$, we say that $f$ is of finite order $\rho(f):=\rho^{*}(f)$.

Proposition 5.11. Let $G=S_{\infty}$ be the infinite symmetric group.

(1) Under condition $(\mathrm{A}), \rho(\Lambda)=0$ (compare with Example $5.9(1.1)$ ).

(2) Assume that $\sigma(k) \asymp\left|G_{k}\right|^{-\gamma}$ for some $\gamma>0$ (condition (A) does not hold!). Then $\rho(\Lambda)=-\gamma$. In particular, for any $\varepsilon>0$ there exist $c_{1}, c_{2}>0$ such that

$$
c_{1} u^{-(\gamma+\varepsilon)} \leq \Lambda(u) \leq c_{2} u^{-(\gamma-\varepsilon)} \quad \text { at } \infty .
$$


Proof of (1). Let $\sigma: \mathbb{R}_{+} \rightarrow \mathbb{R}_{+}$be any continuous decreasing extension of the function $\sigma: \mathbb{Z}_{+} \rightarrow \mathbb{R}_{+}$. By Proposition 5.8,

$$
\Lambda \asymp \sigma \circ \Gamma^{-1}=(\sigma \circ \log ) \circ\left(\exp \circ \Gamma^{-1}\right)=(\sigma \circ \log ) \circ(\Gamma \circ \log )^{-1} .
$$

The function $\sigma \circ \log$ is doubling. Hence for some $k>0$ and $u \gg 1$,

$$
\sigma \circ \log (u) \geq u^{-k} \quad \text { at } \infty
$$

By Stirling's formula, for $u \gg 1$,

$$
(\Gamma \circ \log (u)) \geq(\log u)^{\frac{1}{2} \log u},
$$

and

$$
(\Gamma \circ \log )^{-1}(u) \leq \exp \left(\frac{3 \log u}{\log \log u}\right) .
$$

It follows that for $u \gg 1$,

$$
0 \geq \log (\Lambda(u)) \geq \frac{-3 k \log u}{\log \log u} .
$$

Evidently this inequality implies that $\rho(\Lambda)=0$.

Proof of (2). By Theorem 5.2 and Proposition 5.5,

$$
\frac{1}{\Lambda_{F}} \leq \frac{1}{\Lambda} \leq \frac{1}{\mathcal{T}}
$$

It follows that

$$
\rho_{*}\left(\frac{1}{\Lambda_{F}}\right) \leq \rho_{*}\left(\frac{1}{\Lambda}\right) \leq \rho^{*}\left(\frac{1}{\Lambda}\right) \leq \rho^{*}\left(\frac{1}{\mathcal{T}}\right) .
$$

For $\left|G_{k}\right| \leq u<\left|G_{k+1}\right|$, by Proposition 5.1,

$$
\frac{1}{2} \sigma(k+1)<\mathcal{T}(u)<\sigma(k) .
$$

Hence for such $u$ and $k$, and for some $c_{1}>0$,

$$
\frac{1}{\mathcal{T}(u)} \leq \frac{2}{\sigma(k+1)} \leq c_{1}\left|G_{k+1}\right|^{\gamma}=c_{1}((k+1) !)^{\gamma}
$$

and

$$
\log \frac{1}{\Lambda(u)} \leq \log \frac{1}{\mathcal{T}(u)} \leq c_{1}+\gamma \log (k+1) ! \leq c_{1}+\gamma \log (k+1)+\gamma \log u .
$$

By Stirling's formula $\log k ! \sim k \log k$, hence for any $\varepsilon>0$ there exists $u_{0}>1$ such that for all $u>u_{0}$,

$$
\log \frac{1}{\Lambda(u)} \leq(\gamma+\varepsilon) \log u
$$


This evidently yields the inequality

$$
\rho^{*}\left(\frac{1}{\Lambda}\right) \leq \gamma
$$

By the definition of the function $\Lambda_{F}$, see (5.2),

$$
\frac{1}{\Lambda_{F}(u)}=\left(F^{-1}(u)-1\right)^{2} \text {. }
$$

It follows that

$$
\rho_{*}\left(\frac{1}{\Lambda_{F}(u)}\right)=2 \rho_{*}\left(F^{-1}\right)=\frac{2}{\rho^{*}(F)} .
$$

For $n \leq u \leq n+1$,

$$
F(u) \leq F(n+1)=\left|G_{k(n+1)}\right|=(k(n+1)) !,
$$

where by (5.1),

$$
k(n+1):=\min \left\{k: \lambda_{1}\left(G_{k}\right) \leq(n+1)^{-2}\right\} .
$$

By Proposition 5.3,

$$
\frac{1}{2} \sigma(k)<\lambda_{1}\left(G_{k}\right)<\sigma(k)
$$

hence, for some $c_{2}>0$,

$$
k(n+1) \leq \min \left\{k: \sigma(k) \leq(n+1)^{-2}\right\} \leq \min \left\{k:\left|G_{k}\right| \geq c_{2}(n+1)^{2 / \gamma}\right\}:=\bar{k} .
$$

Evidently, we have

$$
(\bar{k}-1) ! \leq c_{2}(n+1)^{2 / \gamma} \leq \bar{k} !=\bar{k}(\bar{k}-1) ! .
$$

It follows that

$$
F(u) \leq \bar{k} ! \leq c_{2} \bar{k}(n+1)^{2 / \gamma} .
$$

By Stirling's formula, for some $c_{3}, c_{4}>0$,

$$
\bar{k} \leq \bar{k} \log \bar{k} \leq c_{3} \log (\bar{k}-1) ! \leq c_{3} \log c_{2}(n+1)^{2 / \gamma} \leq c_{4} \log (n+1) .
$$

Hence for some $c_{5}>0$ and any $\varepsilon>0$, and $u \gg 1$ we obtain

$$
\log F(u) \leq c_{5}+\log \log (n+1)+\frac{2}{\gamma} \log (n+1) \leq\left(\frac{2}{\gamma}+\varepsilon\right) \log u .
$$

This evidently yields

$$
\rho^{*}(F) \leq \frac{2}{\gamma}
$$

and

$$
\rho_{*}\left(\frac{1}{\Lambda}\right)=\frac{2}{\rho^{*}(F)} \geq \gamma .
$$

The inequalities (5.3) and (5.4) prove the claim. 
Let $\phi: \mathbb{R}_{+} \rightarrow \mathbb{R}_{+}$be any continuous decreasing function such that $\phi(k) \asymp \sigma(k)$ at $\infty$. The following three examples illustrate Proposition 5.11 (1):

(2.1) If $c_{k} \asymp q^{k}, 0<q<1$, then

$$
\Lambda(\tau) \asymp(\phi \circ \log ) \circ(\Gamma \circ \log )^{-1}(\tau) \asymp\left((\Gamma \circ \log )^{-1}(\tau)\right)^{-A},
$$

where $A=\log \frac{1}{q}$. Applying Stirling's formula, we obtain

$$
\log \frac{1}{\Lambda(\tau)} \sim \frac{A \log \tau}{\log \log \tau} \quad \text { at } \infty
$$

(2.2) If $c_{k} \asymp k^{-p}, p>1$, then

$$
\Lambda(\tau) \asymp\left(\phi \circ \Gamma^{-1}\right)(\tau) \asymp\left(\frac{\log \tau}{\log \log \tau}\right)^{-p+1} \quad \text { at } \infty .
$$

(2.3) If $c_{k} \asymp 1 /\left(k \cdot \log k \cdot \log _{(2)} k \cdots\left(\log _{(n)} k\right)^{p}\right), p>1$, then

$$
\Lambda(\tau) \asymp\left(\phi \circ \Gamma^{-1}\right)(\tau) \asymp\left(\log _{(n+1)} \tau\right)^{1-p} \text { at } \infty \text {. }
$$

\section{Spectral distribution and return probability}

Let $\lambda \rightarrow E_{\lambda}$ be the spectral resolution of a Laplacian $\Delta$, with spectral distribution function $N(\lambda)$. On finitely generated groups, and for symmetric probability measures with generating supports and finite second moments, the (dilatational equivalence class of the) function $N$ is stable under quasi-isometry. See [4], [23], [22], 0.2.C. Under mild regularity assumptions, $\Lambda$ and $N$ are related by the formula

$$
N(\lambda) \stackrel{d}{\simeq} \frac{1}{\Lambda^{-1}(\lambda)}
$$

Proposition 6.1. Under condition (A) the following properties hold.

$$
N \stackrel{d}{\simeq} \frac{1}{\mathcal{T}^{-1}} \quad \text { at } 0 \text { and } \Lambda \asymp \mathcal{T} \quad \text { at } \infty
$$

Proof. The second equivalence of (6.1) follows from Theorem 5.7. To prove first observe that for $\sigma(k+1) \leq u<\sigma(k)$,

$$
N(u)=\frac{1}{\left|G_{k+1}\right|} .
$$

Also, by Proposition 5.1(4), for $\left|G_{k}\right| \leq \tau \leq\left|G_{k+1}\right|$,

$$
\frac{1}{2} \sigma(k+1)<\mathcal{T}(\tau)<\sigma(k) .
$$


Define $\theta_{1}:=\mathcal{T}\left(\left|G_{k+1}\right|\right)$ and $\theta_{2}:=\mathcal{T}\left(\left|G_{k}\right|\right)$. Then

$$
\frac{1}{2} \sigma(k+1)<\theta_{1}<\theta_{2}<\sigma(k) .
$$

Observe that the condition (A) implies that there exists a constant $\lambda>0$ such that

$$
\sigma(k+1)>\frac{1}{1+\lambda} \sigma(k), \quad k=0,1, \ldots
$$

Putting all these facts together and the fact that $\theta \rightarrow \mathcal{T}^{-1}(\theta)$ decreases, we obtain that for $\sigma(k+1) \leq u<\sigma(k)$,

$$
\begin{aligned}
\frac{1}{N(u)} & =\left|G_{k+1}\right| \\
& =\mathcal{T}^{-1}\left(\theta_{1}\right)>\mathcal{T}^{-1}(\sigma(k))>\mathcal{T}^{-1}((1+\lambda) \sigma(k+1))>\mathcal{T}^{-1}((1+\lambda) u),
\end{aligned}
$$

and

$$
\begin{aligned}
\frac{1}{N(u)} & =\left|G_{k+1}\right| \\
& =\mathcal{T}^{-1}\left(\theta_{1}\right)<\mathcal{T}^{-1}\left(\frac{1}{2} \sigma(k+1)\right)<\mathcal{T}^{-1}\left(\frac{1}{2(1+\lambda)} \sigma(k)\right)<\mathcal{T}^{-1}\left(\frac{u}{2(1+\lambda)}\right) .
\end{aligned}
$$

The proof is finished.

Example 6.2. Let $G=\bigcup_{n=0}^{\infty} G_{n}$ and $v: \mathbb{R}_{+} \rightarrow \mathbb{R}_{+}$be the volume function, that is $v(k)=\left|G_{k}\right|, k=0,1, \ldots$ Let $\phi: \mathbb{R}_{+} \rightarrow \mathbb{R}_{+}$be any continuous decreasing function such that $\phi(k) \asymp \sigma(k)$ at $\infty$. Then, according to Proposition 5.8 and Proposition 6.1, under condition (A),

$$
N \stackrel{d}{\simeq} \frac{1}{v \circ \phi^{-1}}
$$

1) Let $G=\bigcup_{k}(\mathbb{Z} / \mathbb{Z})^{k}$. Then $v(x)=2^{x}$, and the formula for $N$ takes the form

$$
N(u) \stackrel{d}{\asymp} \exp \left(-\frac{1}{\gamma} \phi^{-1}(u)\right), \quad \text { where } \gamma=1 / \log 2 .
$$

In particular, we obtain the following estimates.

(1.1) If $c_{k} \asymp q^{k}, 0<q<1$, then $N(u) \asymp u^{1 / A \gamma}$ at 0 , where $A=\log \frac{1}{q}$ and $\gamma=\frac{1}{\log 2}$.

(1.2) If $c_{k} \asymp k^{-p}, p>1$, then $N(u) \stackrel{d}{\simeq} \exp \left\{-u^{\frac{1}{1-p}}\right\}$ at 0 .

(1.3) If $c_{k} \asymp 1 /\left(k \cdot \log k \cdot \log _{(2)} k \cdots\left(\log _{(n)} k\right)^{p}\right), p>1$, then we have $N(u) \stackrel{d}{\simeq}$ $\exp \left\{-\exp _{(n)}\left(u^{\frac{1}{1-p}}\right)\right\}$ at 0 . 
2) Let $G=S_{\infty}$ be the infinite symmetric group endowed with its volume function $v(x)=\Gamma(1+x)$, that is $G_{n}=S_{n}$ and $\left|G_{n}\right|=n$ !.

(a) Assume that condition (A) holds. Then the formula for the function $N$ has the form

$$
\log \frac{1}{N(u)} \stackrel{d}{\simeq}(\log \Gamma) \circ \phi^{-1}(u) .
$$

Stirling's formula and straightforward computations give the following results.

(2.1) If $c_{k} \asymp q^{k}, 0<q<1$, then with $A=\log \frac{1}{q}$

$$
\log \frac{1}{N(u)} \sim \frac{1}{A}\left(\log \frac{1}{u}\right)\left(\log \log \frac{1}{u}\right) \quad \text { at } 0 .
$$

(2.2) If $c_{k} \asymp k^{-p}, p>1$, then

$$
N(u) \stackrel{d}{\simeq} \exp \left(-\left(\frac{1}{u}\right)^{\frac{1}{p-1}} \log \frac{1}{u}\right) \quad \text { at } 0 .
$$

(2.3) If $c_{k} \asymp 1 /\left(k \cdot \log k \cdot \log _{(2)} k \cdots\left(\log _{(n)} k\right)^{p}\right), p>1$, then

$$
N(u) \stackrel{d}{\simeq} \exp \left(-\exp _{(n)}\left(\frac{1}{u}\right)^{\frac{1}{p-1}}\right) \text { at } 0 .
$$

(b) Assume that $\sigma(k) \asymp\left|G_{k}\right|^{-\gamma}$ for some $\gamma>0$. We claim that in this case $N(u)$ is of finite order $1 / \gamma$ at zero, that is, $u \rightarrow 1 / N(1 / u)$ is of finite order $1 / \gamma$ at infinity. In other words, for any $\varepsilon>0$ there exist $c_{1}, c_{2}>0$ such that

$$
c_{1} u^{\frac{1}{\gamma}+\varepsilon} \leq N(u) \leq c_{2} u^{\frac{1}{\nu}} \quad \text { at } 0 .
$$

Indeed, let $\sigma(k+1) \leq u<\sigma(k)$. By assumption, for some $c_{1}>0$,

$$
N(u)=\frac{1}{\left|G_{k+1}\right|}=\frac{1}{(k+1) !} \leq\left(\frac{u}{c_{1}}\right)^{1 / \gamma} .
$$

It follows that

$$
\rho_{*}(N):=\liminf _{\lambda \rightarrow \infty} \frac{\log \frac{1}{N(1 / \lambda)}}{\log \lambda} \geq \frac{1}{\gamma} .
$$

On the other hand, by assumption,

$$
N(u)=\frac{1}{(k+1) !}=\frac{(k !)^{-1}}{k+1} \geq\left(\frac{u}{c_{2}}\right)^{1 / \gamma} \frac{1}{k+1} .
$$

By Stirling's formula, for any $\varepsilon>0$ and for all $k \geq k(\varepsilon)>1$,

$$
k+1 \leq k \log k \leq(1+\varepsilon) \log k ! \leq \frac{1+\varepsilon}{\gamma} \log \frac{c_{2}}{u} \leq \frac{1+2 \varepsilon}{\gamma} \log \frac{1}{u} .
$$


It follows that for some $c_{3}>0$,

$$
N(u) \geq \frac{c_{3} u^{1 / \gamma}}{\log \frac{1}{u}}
$$

This inequality shows that

$$
\rho^{*}(N):=\limsup _{\lambda \rightarrow \infty} \frac{\log \frac{1}{N(1 / \lambda)}}{\log \lambda} \leq \frac{1}{\gamma} .
$$

The claim is proved.

Let $\left(\mu_{t}\right)_{t>0}$ be a weakly continuous convolution semigroup of probability measures on $G$ associated with the measure $\mu=\mu(c)$, that is, $\left.\mu_{t}\right|_{t=1}=\mu$ (see Proposition 3.2). Let $P$ and $\Delta=P-I$ be the corresponding transition operator and the corresponding Laplacian.

Definition 6.3. Define the return probability function $p(t)$ as follows:

$$
p(t):=\left(\delta_{e} * \mu_{t}, \delta_{e}\right)=\left(P^{t} \delta_{e}, \delta_{e}\right)
$$

By spectral theory,

$$
p(t)=\left((I+\Delta)^{t} \delta_{e}, \delta_{e}\right)=\int_{0}^{\infty}(1-\lambda)^{t} d N(\lambda) .
$$

Our first observation is that

$$
p(t) \stackrel{d}{\simeq} \int_{0}^{\infty} e^{-\lambda t} d N(\lambda)
$$

Writing the function $t \rightarrow p(t)$ in the form

$$
p(t)=\exp (-t \cdot R(t)), \quad t>0,
$$

we observe that since the group $G$ is amenable, $R(t)=o(1)$ at $\infty$.

Proposition 6.4. Let $v, \phi: \mathbb{R}_{+} \rightarrow \mathbb{R}_{+}$be two continuous monotone functions such that $v(k) \asymp\left|G_{k}\right|$ and $\phi(k) \asymp \sigma(k), k \in \mathbb{Z}_{+}$. Then under condition (A),

$$
R \stackrel{d}{\asymp}\left(\frac{(\log v) \circ \phi^{-1}}{\mathrm{id}}\right)^{-1}
$$

Proof. Using first Proposition 2.2, and then Proposition 6.1 and Proposition 5.8, we can write

$$
\log \frac{1}{p} \sim \mathscr{L}\left(\log \frac{1}{N}\right) \stackrel{d}{\simeq} \mathscr{L}\left((\log v) \circ \phi^{-1}\right) \asymp \mathrm{d} \cdot\left(\frac{(\log v) \circ \phi^{-1}}{\mathrm{id}}\right)^{-1} .
$$

This evidently gives the desired result. 
Example 6.5. 1) Let $G=(\mathbb{Z} / 2 \mathbb{Z})^{(\infty)}$. Choosing $v(x)=2^{x}$, the formula for $p(t)$ from Proposition 6.4 takes the form

$$
p(t)=\exp (-t R(t)), \quad R(t) \asymp\left(\frac{d}{\mathrm{id}}\right)^{-1} .
$$

In particular we obtain the following estimates.

(1.1) If $c_{k} \asymp q^{k}, 0<q<1$, then $N(\lambda) \asymp \lambda^{1 / A \gamma}$ at 0 , where $A=\log \frac{1}{q}$ and $\gamma=1 / \log 2$ (see Example 6.2(1)). Hence, a standard Laplace transform argument gives

$$
p(t) \stackrel{d}{\simeq} \int_{0}^{\infty} e^{-\lambda t} d N(\lambda) \asymp t^{-1 / A \gamma} \quad \text { at } \infty .
$$

(1.2) If $c_{k} \asymp k^{-p}, p>1$, then $R(t) \asymp t^{-\frac{p-1}{p}}, p(t) \stackrel{d}{\simeq} \exp \left(-t^{\frac{1}{p}}\right)$ at $\infty$.

(1.3) If $c_{k} \asymp 1 /\left(k \cdot \log k \cdot \log _{(2)} k \cdots\left(\log _{(n)} k\right)^{p}\right)$ and $p>1$, whence in this case we claim that $R \asymp \phi$. Indeed, one can easily show that under the assumption $x \rightarrow(\phi \circ \exp )(x)$ is doubling,

$$
\left(\frac{\phi^{-1}}{\mathrm{id}}\right)^{-1} \asymp \phi, \quad p(t) \stackrel{d}{\simeq} \exp \left(-\frac{t}{\left(\log _{(n)} t\right)^{p-1}}\right) \quad \text { at } \infty .
$$

2) Let $G=S_{\infty}$. In this case $v(x)=\Gamma(1+x), \log v(x) \sim x \log x$ and, assuming that the condition (A) holds, the formula for $p(t)$ takes the following form:

$$
p(t)=\exp (-t R(t)), \quad R(t) \stackrel{d}{\asymp}\left(\frac{\phi^{-1} \log \phi^{-1}}{\mathrm{id}}\right)^{-1} .
$$

(2.1) If $c_{k} \asymp q^{k}, 0<q<1$, whence

$$
\log \frac{1}{N(u)} \sim \frac{1}{A} \log \frac{1}{u} \log \log \frac{1}{u} \quad \text { at } 0
$$

and

$$
\log \frac{1}{p(t)} \sim \frac{1}{A}(\log t)(\log \log t) \quad \text { at } \infty .
$$

(2.2) If $c_{k} \asymp k^{-p}$ and $p>1$, then

$$
R(t) \asymp\left(\frac{\log t}{t}\right)^{1-\frac{1}{p}} \text { and } \quad p(t) \stackrel{d}{\simeq} \exp \left(-t^{\frac{1}{p}}(\log t)^{1-\frac{1}{p}}\right) \quad \text { at } \infty \text {. }
$$

(2.3) Let $c_{k} \asymp k^{-1} /\left(\log k \cdot \log _{(2)} k \cdots\left(\log _{(n)} k\right)^{p}\right), p>1$, then

$$
R(t) \asymp \phi(t)=\left(\log _{(n)} t\right)^{-p+1} \text { at } \infty
$$

and

$$
p(t) \stackrel{d}{\simeq} \exp \left(-\frac{t}{\left(\log _{(n)} t\right)^{p-1}}\right) \quad \text { at } \infty
$$


(2.4) Assume that $\sigma(k) \asymp\left|G_{k}\right|^{-\gamma}$ for some $\gamma>0$. Then the function $p(t)$ is offinite order $-1 / \gamma$. Indeed, $p(t)$ and $N(\lambda)$ are related by (6.2). Standard Laplace transform argument yield the result. In particular, for any $\varepsilon>0$ there exist $c_{1}, c_{2}>0$ such that

$$
c_{2} t^{-\frac{1}{\gamma}-\varepsilon} \leq p(t) \leq c_{1} t^{-\frac{1}{\gamma}} \quad \text { at } \infty
$$

Some particular results based on the computations from above are presented in the following table. Some comments about the table are in order. Given a measure $\mu(c)$ on the locally finite group $\bigcup_{k}(\mathbb{Z} / 2 \mathbb{Z})^{k}$, or $\bigcup_{k} S_{k}$, the first, second, third, and fourth column, shows respectively, the tail $\sigma(k)$, the isospectral profile $\Lambda(\tau)$, the spectral density $N(u)$, and the return probability $p(t)$, associated to the measure $\mu(c)$. In all given examples, the asymptotic behavior of any of the function determines the asymptotic behaviors of the three others. In other words, the information given in any of the column, completely determines the information given in the three other columns. Notice that the asymptotic behavior of the coefficients $c_{k}$ defining $\mu(c)$ cannot be recovered from the asymptotic behavior of the tail $\sigma(k)=\sum_{i>k} c_{k}$, not even in the special cases considered in the table. They can be recovered if some regularity assumptions from Karamata theory are made about the $c_{k}$ (for example monotonicity). Notice that the $c_{k}$ are not necessary monotone in our constructions.

Acknowledgments. The paper was written during stays at the Laboratoire Poncelet in Moscow (UMI 2615) and at the Steklov Mathematical Institute. We are grateful to M. Tsfasman and to A. Shiryaev for their invitations. We thank A. Erschler, Y. Guivarc'h, V. Kaimanovich, L. Saloff-Coste, A. Vershik and W. Woess for fruitful discussions and valuable comments. 


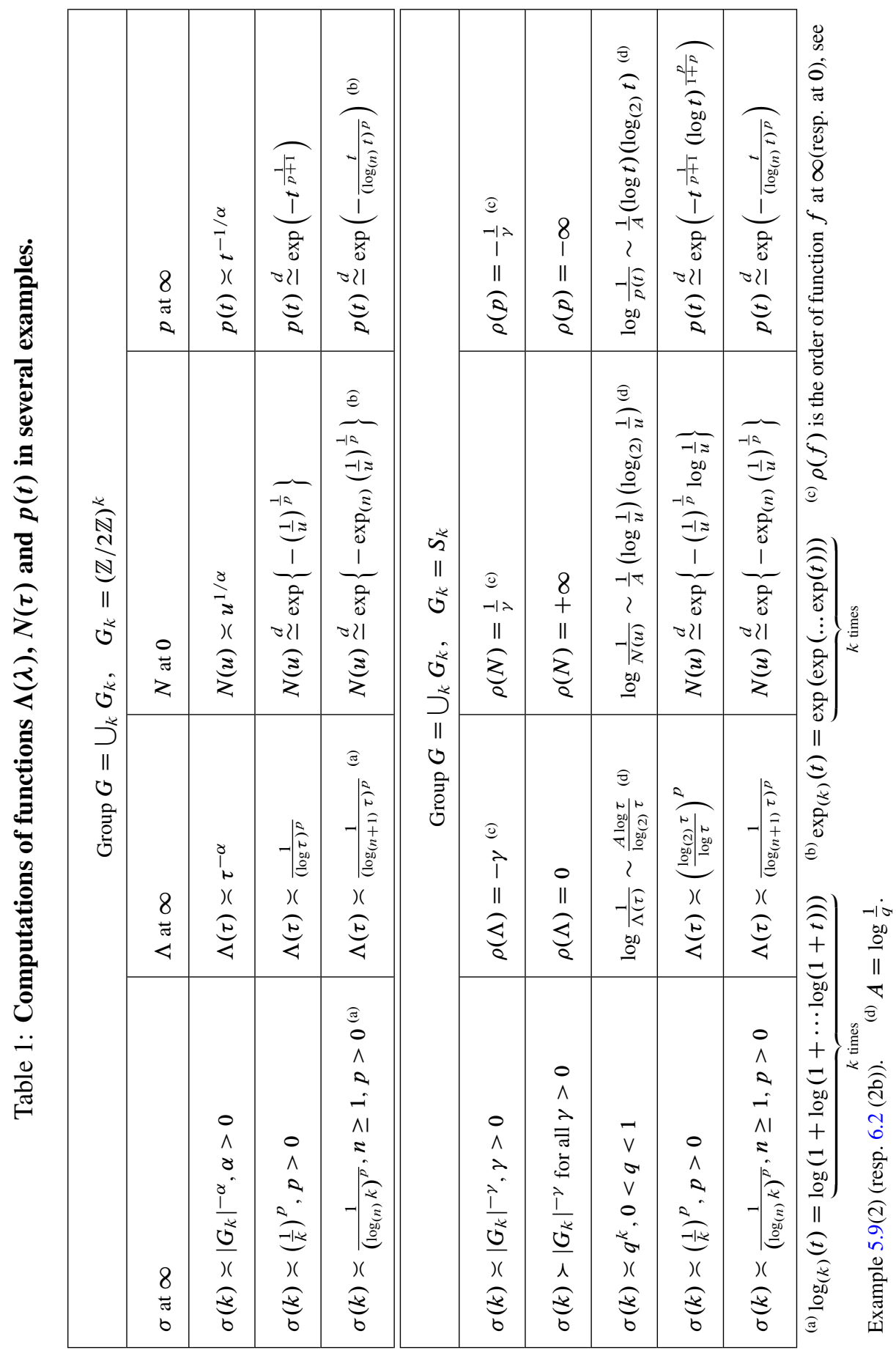




\section{References}

[1] D. Bakry, T. Coulhon, M. Ledoux, and L. Saloff-Coste, Sobolev inequalities in disguise. Indiana Univ. Math. J. 44 (1995), 1033-1074. Zbl 0857.26006 MR 1386760

[2] A. Bendikov and B. Bobikau, Long time behavior of random walks on abelian groups. Colloq. Math. 118 (2010), 445-464. Zbl 1196.60079 MR 2602160

[3] A. Bendikov, T. Coulhon, and L. Saloff-Coste, Ultracontractivity and embedding into $L^{\infty}$. Math. Ann. 337 (2007), 817-853. Zbl 1132.47031 MR 2285739

[4] A. Bendikov, C. Pittet, and R. Sauer, Spectral distribution and $L^{2}$-isoperimetric profile of Laplace operators on groups. Math. Ann. 354 (2012), 43--72. Zbl 1268.43001 MR 2957618

[5] A. Bendikov and L. Saloff-Coste, Random walks on groups and discrete subordinations. Math. Nachr. 285 (2012), 580-605. Zbl 06034377 MR 2902834

[6] C. Berg and J. P. R. Christensen, Sur la norme des opérateurs de convolution. Invent. Math. 23 (1974), 173-178. Zbl 0261.22009 MR 0338685

[7] S. Brofferio and W. Woess, On transience of card shuffling. Proc. Amer. Math. Soc. 129 (2001), 1513-1519. Zbl 0964.60058 MR 1709741

[8] G. Carron, Inégalités isopérimétriques de Faber-Krahn et conséquences. In Actes de la Table Ronde de Géométrie Différentielle (Luminy, 1992), Sémin. Congr. 1, Soc. Math. France, Paris 1996, 205-232. Zbl 0884.58088 MR 1427759

[9] T. Coulhon, Ultracontractivity and Nash type inequalities. J. Funct. Anal. 141 (1996), 510-539. Zbl 0887.58009 MR 1418518

[10] T. Coulhon, A. Grigor'yan, and D. Levin, On isoperimetric profiles of product spaces. Comm. Anal. Geom. 11 (2003), 85-120. Zbl 1085.53027 MR 2016197

[11] T. Coulhon, A. Grigor'yan, and C. Pittet, A geometric approach to on-diagonal heat kernel lower bounds on groups. Ann. Inst. Fourier (Grenoble) 51 (2001), 1763-1827. Zbl 1137.58307 MR 1871289

[12] D. A. Darling and P. Erdős, On the recurrence of a certain chain. Proc. Amer. Math. Soc. 19 (1968), 336-338. Zbl 0164.47502 MR 0222962

[13] J. Dodziuk, Difference equations, isoperimetric inequality and transience of certain random walks. Trans. Amer. Math. Soc. 284 (1984), 787-794. Zbl 0512.39001 MR 743744

[14] R. M. Dudley, Random walks on abelian groups. Proc. Amer. Math. Soc. 13 (1962), 447-450. Zbl 0106.12403 MR 0141167

[15] A. Erschler, Isoperimetry for wreath products of Markov chains and multiplicity of selfintersections of random walks. Probab. Theory Related Fields 136 (2006), 560-586. Zbl 1105.60009 MR 2257136

[16] W. Feller, An introduction to probability theory and its applications. Vol. I, 3rd ed., John Wiley \& Sons, New York 1968. Zbl 0155.23101 MR 0228020

[17] W. Feller, An introduction to probability theory and its applications. Vol. II, 2nd ed., John Wiley \& Sons, New York 1971. Zbl 0219.60003 MR 0270403

[18] N. Fereı̆g and S. A. Molčanov, Random walks on Abelian groups with an infinite number of generators. Vestnik Moskov. Univ. Ser. I Mat. Mekh. 1978 (1978), 22-29; English transl. Moscow Univ. Math. Bull. 33 (1978), No. 5, 17-23. Zbl 0411.60073 MR 516017 
[19] L. Flatto and J. Pitt, Recurrence criteria for random walks on countable Abelian groups. Illinois J. Math. 18 (1974), 1-19. Zbl 0296.60045 MR 0341616

[20] A. A. Grigor' yan, The heat equation on noncompact Riemannian manifolds. Mat. Sb. 182 (1991), No. 1, 55-87; English transl. Math. USSR-Sb. 72 (1992), 47-77. Zbl 0776.58035 MR 1098839

[21] A. Grigor' yan, Heat kernel upper bounds on a complete non-compact manifold. Rev. Mat. Iberoamericana 10 (1994), 395-452. Zbl 0810.58040 MR 1286481

[22] M. Gromov, Geometric group theory (Sussex, 1991), vol. 2: Asymptotic invariants of infinite groups. London Math. Soc. Lecture Note Ser. 182, Cambridge University Press, Cambridge 1993. Zbl 0841.20039 MR 1253544

[23] M. Gromov and M. A. Shubin, Von Neumann spectra near zero. Geom. Funct. Anal. 1 (1991), 375-404. Zbl 0751.58039 MR 1132295

[24] E. Hewitt and K. A. Ross, Abstract harmonic analysis. Vol. I, 2nd ed., Grundlehren Math. Wiss. 115, Springer-Verlag, Berlin 1979. Zbl 0416.43001 MR 0551496

[25] M. A. Kasymdzhanova, Recurrence of invariant Markov chains on a class of abelian groups. Vestnik Moskov. Univ. Ser. I Mat. Mekh. 1981 (1981), 3-7; English transl. Moscow Univ. Math. Bull. 36 (1981), No. 3, 1-6. Zbl 0486.60013 MR 641203

[26] H. Kesten, Full Banach mean values on countable groups. Math. Scand. 7 (1959), 146-156. Zbl 0092.26704 MR 0112053

[27] G. F. Lawler, Recurrence and transience for a card shuffling model. Combin. Probab. Comput. 4 (1995), 133-142. Zbl 0837.60062 MR 1342857

[28] W. Lück, $L^{2}$-invariants: theory and applications to geometry and $K$-theory. Ergeb Math. Grenzgeb. (3) 44, Springer-Verlag, Berlin 2002. Zbl 1009.55001 MR 1926649

[29] C. Pittet, The isoperimetric profile of homogeneous Riemannian manifolds. J. Differential Geom. 54 (2000), 255-302. Zbl 1035.53069 MR 1818180

[30] C. Pittet and L. Saloff-Coste, Amenable groups, isoperimetric profiles and random walks. In Geometric group theory down under (Canberra 1996), Walter de Gruyter, Berlin 1999, 293--316. Zbl 0934.43001 MR 1714851

[31] C. Pittet and L. Saloff-Coste, On the stability of the behavior of random walks on groups. J. Geom. Anal. 10 (2000), 713-737. Zbl 0988.60517 MR 1817783

[32] C. Pittet and L. Saloff-Coste, Random walks on finite rank solvable groups. J. Eur. Math. Soc. (JEMS) 5 (2003), 313-342. Zbl 1057.20026 MR 2017850

[33] D. Revuz, Markov chains. 2nd ed., North-Holland Math. Library 11, North-Holland Publishing Co., Amsterdam 1984. Zbl 0539.60073 MR 758799

[34] R. T. Rockafellar and R. Tyrrell, Convex analysis. Princeton Landmarks in Mathematics, Princeton University Press, Princeton, NJ, 1997. Zbl 0932.90001 MR 1451876

[35] L. Saloff-Coste, Sur la décroissance des puissances de convolution sur les groupes. Bull. Sci. Math. (2) 113 (1989), 3-21. Zbl 0682.43004 MR 984425

[36] F. Spitzer, Principles of random walk. 2nd ed., Graduate Texts in Math. 34, SpringerVerlag, New York 1976. Zbl 0979.60002 MR 0388547 
Received June 23, 2011; revised March 10, 2012
A. Bendikov, Institute of Mathematics, Wrocław University, 50-384 Wrocław, Pl. Grunwaldzki 2/4, Poland
E-mail: bendikov@math.uni.wroc.pl
B. Bobikau, Institute of Mathematics, Wrocław University, 50-384 Wrocław, Pl. Grunwaldzki 2/4, Poland
E-mail: bobikau@math.uni.wroc.pl
C. Pittet, I2M, Aix-Marseille Université, 39 rue Frédéric Joliot-Curie, 13453 Marseille Cedex 13, France
E-mail: pittet@math.cnrs.fr 